\title{
Synthesis and Characterization of Zinc (II) Phthalocyanine for Screening Potential Solar Cell Dye Application
}

\author{
Connor Atkinson \\ Department of Chemistry, University of Manchester \\ * Corresponding author email: Connoratkinsonlad@gmail.com \\ Received: 2 December 2021 / Revised: 16 December 2021 / Accepted: 05 January 2022 / Published: 12 January 2022
}

\begin{abstract}
Phthalocyanine molecules have the potential to be used in select Dye Sensitized Solar Cells (DSSCs) and Luminescent Solar Concentrators (LSCs), due to UV-Vis absorbance in the 300-450 (nm) Soret Band, corresponding to $\pi$ HOMO-1 to $\pi^{*}$ LUMO transition and 550-690 (nm) Q-band, corresponding to $\pi$ HOMO to $\pi^{*}$ LUMO transitions. In this study Tetranitro Zinc (II) Phthalocyanine is synthesized via base catalysis before the product is characterized via IR, 1H NMR \& UV-Vis analysis. Assessing the desirability of the Tetranitro Zinc (II) Phthalocyanine as a solar organic semiconducting dye in DSSCs and LSCs. The desirability is assessed by novel computational DFT calculations, of the aggregation binding mode to deduce if Aggregation-Caused Quenching (ACQ) is occurring in the aggregated sample. ACQ is known to reduce DSSCs and LSCs generation of useful photo-active current. Aggregation-Caused Quenching (ACQ) is mathematically indicated in Phthalocyanine aggregation and Tetranitro Zinc (II) Phthalocyanine's desirability is assessed for further use in DSSCs and LSCs.
\end{abstract}

Keywords: Phthalocyanine, DFT, Dye Aggregation, Aggregation-Caused Quenching.

\section{Introduction}

Unsaturated hydrocarbons are neighbouring $\mathrm{SP}^{2}$ hybridised carbon atoms, arranged linearly. Neighbouring $\mathrm{P}_{(z)}$ orbitals interact forming a new $\pi$ bond, as 2 new molecular orbitals HOMO and LUMO form. The HOMO $\pi$-bond corresponds to 2 converged $\mathrm{P}_{(z)}$ orbital wavefunctions. The LUMO $\pi^{*}$-antibonding bond corresponds to 2 non-converged $\mathrm{P}_{(z)}$ orbital wavefunctions. As the number of neighbouring $\mathrm{P}_{(z)}$ orbitals increases, the number of orbital interactions increases, decreasing the band gap energy between HOMO, conduction band, and LUMO, valance band, frontier orbitals. Electrons will transition from their ground state energy, to an allowed excited state $\pi$ to $\pi^{*}$, and back, as electromagnetic radiation is absorbed. The wavelength of absorbed light in (nm) will correspond to the exited photon's new energy state in (eV). With an electron being excited to the LUMO a hole must also be formed at the HOMO, resulting is a bound electron-hole pair.

A Luminescent Solar Concentrator (LSC) consists of; Dye molecules doped into a transparent matrix material. Upon excitation \& relaxation of the ye's frontier electron, the emitted photonic energy becomes wavelength stoke shifted and trapped in the host matrix via internal reflection thus it's guided towards the sides of the device. The concentrated photonic energy at the sides of the slab can then be converted into current via solar cells at the sides of the slab. A Dye Sensitized Solar Cell (DSSC) consists of; Dye molecules adsorbed onto an electron transporting material. Upon excitation of the dye's frontier electron, the conducting electron becomes oxidized and trapped in the conducting surface thus forming a separated electron-hole pair. The electrons can then do work, via an electrical circuit, before being transferred to the electrolyte at the cathode and recombining with the dye molecule hole.

Organic semiconducting dyes are vital to the development of emerging solar devices, such as Dye Sensitised Solar Cells (DSSCs) and luminescent Solar Concentrators (LSCs), as they yield conducting electrons and current [1,2]. Dye Sensitized Solar Cells contain an organic semiconducting dye adsorbed onto a porous 
Synthesis and Characterization of Zinc (II) Phthalocyanine for Screening Potential Solar Cell Dye Application

electron transporting material. [1,2] Low solubility of the organic semiconducting dye, at high concentrations, can lead to organic semiconducting dye aggregation absorbance onto the electron transporting material. Luminescent Solar Concentrators contain an organic semiconducting dye, as a lumophore, doped in a matrix [3,4]. Low solubility of the organic semiconducting dye with the matrix, at high concentrations, can lead to organic semiconducting dye aggregation in the matrix. Both aggregation in Dye Sensitized Solar Cells as well as Luminescent Solar Concentrators typically provides undesirable effects such as aggregation-caused quenching (ACQ), lowering solar cell device performance such as Short Circuit Current and Open Circuit Voltage [1,2]. This effect is very common for organic semiconducting dyes, theorised but not yet proven to result from $\mathrm{p}-\mathrm{p}$ stacking interactions causing aggregation and thus aggregation-caused quenching $[5,6]$.

Phthalocyanine is an organic semiconducting dye that's notoriously insoluble, theorised to result from its conjugated $\pi$ framework allowing $\mathrm{p}-\mathrm{p}$ orbital stacking interactions to from between two Phthalocyanine molecules. Phthalocyanine isn't normally desirable as a solar organic semiconducting dye, theorised to result from this aggregation limiting solar cell efficiencies. Many variations to phthalocyanines structures can be introduced however, to alter its ability to aggregate and thus altering its theorised aggregation-caused quenching [7,8]. Variations can potentially make Phthalocyanine more desirable as a solar organic semiconducting dye. Various phthalocyanine organic semiconducting dye structures have been created, each possessing different degrees of aggregation, by simply reacting unique phthalonitrile starting monomer structures together. Phthalocyanine aggregation might be limited, transformed or induced via various structural changes.

Herein, we shall investigate the tetranitro Zinc Phthalocyanine variation. Assessing how the tetranitro Zinc structural variations, influence the organic semiconducting dyes aggregation $[7,9]$.

Comparing UV-Vis data against DFT computed data, allowing the type of aggregation occurring between the two tetranitro Zinc Phthalocyanine variation molecules to be deduced as well as supporting the theorised cause of aggregation-caused quenching theory. Allowing the desirability of the solar organic semiconducting dye to be screened for application in further studies.

\section{Research Methodology}

All reagents and solvents, unless stated otherwise, were purchased from Sigma Aldrich Chemicals and were used without further purification.

\subsection{Tetranitro Zinc (II) Phthalocyanine Synthesis}

Tetranitro Zinc (II) Phthalocyanine was synthesised via the following synthetic methodology.

0.34(g), 1.964(mmol) 4-Nitrophthalonitrile, was added into 5(mL) 1-Pentanol, in a 3-neck round bottom flask, fitted with a reflux condenser and magnetic stir bar, closing all openings with 3 rubber septa. The flask and reaction mixture were purged with nitrogen gas using a nitrogen gas filled balloon, fitted with a long needle submerged into the reaction solution.

After purging the solution for 15 minutes, $0.125(\mathrm{~g}), 0.92(\mathrm{mmol})$ zinc chloride was added under nitrogen overpressure, followed by addition of $0.21(\mathrm{~mL}) \mathrm{DBU}$. The solution was then further purged with nitrogen gas for 10 minutes, to ensure inert nitrogen atmosphere had been achieved. The reaction mixture was then heated to $150\left({ }^{\circ} \mathrm{C}\right)$ for $15(\mathrm{~h})$ under $1(\mathrm{~atm})$ nitrogen atmosphere, by attaching a nitrogen balloon to the top of the condenser, imitating the reaction.

The mixture was cooled to room temperature for 10 minutes, then $20(\mathrm{~mL})$ of methanol was added to the solution before cooling over an ice bath, allowing the Tetranitro Zinc (II) Phthalocyanine crude product to precipitate out of solution. The crude product was washed to remove synthesis impurities. The dark blue to dark green product was then obtained by vacuum Buchner filtration and washed sequentially with Ethanol, Methanol, water, $5 \%$ hydrogen chloride solution and water, utilising $5(\mathrm{~mL})$ of each solvent leaving a refined final product with no impurities. 


\subsection{Characterization Equipment}

FT-IR and ATR-IR spectroscopy were completed using, Spectrum 100 (Perkin Elmer)/Alpha (Bruker), (Specac Goldengate/ embedded platinum) with a scan range of $550-4000\left(\mathrm{~cm}^{-1}\right)$, wavenumbers are reported in $\mathrm{cm}^{-1}$. 1H NMR spectra were recorded using, $500 \mathrm{MHz}$ Bruker Advance III HD NMR spectrometer (equipped with a broadband ambient probe). UV-Vis spectroscopy were carried out using a Analytik Jena Specord 50 Spectrophotometer scan range 190-1100(nm), wavelengths are reported in nm. TD-DFT are completed with Orca 4.2.1, in good accordance with the orca user manual and best practices for Phthalocyanine molecules. Input files are created utilizing: BP86/def2-SVP optimized geometries for all atoms but Metals were def2-TZVP was chosen, both applying RIJCOSX auxiliary basis set and Tight-SCF fitting parameters.

\subsection{Tetranitro Zinc (II) Phthalocyanine Characterisation}

The solid product was vacuum and oven dried. $0.2679(\mathrm{~g}), 0.3534(\mathrm{mmol})$ of product was obtained, a $72 \%$ yield, and characterised via solid-state ATR-IR, NMR and UV-Vis spectroscopy, to deduce if the reaction had successfully yielded Tetranitro Zinc (II) Phthalocyanine and deduce the how the tetranitro Zinc structural variations, influence the organic semiconducting dyes aggregation.

The sparingly soluble NMR sample was filtered thought cotton before analysis, limiting particulate concentration and thus aggregation to measure reduced aggregated samples. High concentration UV-Vis samples were created, at 0.00009011(M), 0.0001759(M), 0.0002577(M) \& 0.0003358(M) concentrations, inducing aggregation to measure induced aggregated samples.

ATR-IR spectra is show in table 1,1H NMR spectra is shown in table 2, UV-Vis spectra is shown if figure 1 and all important data is discussed in associated text. All computational data is produced for comparison of experimental data, with pure gas phase DFT calculated data and to support aggregation-caused quenching theory via calculated p-p bonding.

\section{Results and Discussion}

\subsection{ATR-IR Results}

The ATR-IR plot of Tetranitro Zinc (II) Phthalocyanine product analysis was produced and is presented in Table 1.

Table 1: Tetranitro Zinc (II) Phthalocyanine ATR-IR spectra data

\begin{tabular}{|c|c|}
\hline Wavenumber $\left(\mathrm{cm}^{-1}\right)$ & Assignment \\
\hline 1518 & $\mathrm{NO}_{2}$ \\
\hline 1338 & $\mathrm{NO}_{2}$ \\
\hline 3420 & Aromatic C-H \\
\hline 1090 & $\mathrm{C}=\mathrm{C}$ \\
\hline 1602 & $\mathrm{C}=\mathrm{N}$ \\
\hline 725 & C-N-C \\
\hline
\end{tabular}

Table 1 indicates the formation of Tetranitro Zinc (II) Phthalocyanine with 1518(cm-1)/1338( $\left.\mathrm{cm}^{-1}\right)$ nitro group and 3420(cm-1)/1090(cm-1)/1602/725(cm-1)/696(cm-1) Phthalocyanine metal organic framework, observed.[10]

\subsection{H NMR Results}

A low aggregation $1 \mathrm{H}$ NMR spectra of Tetranitro Zinc (II) Phthalocyanine product was produced and is presented in Table 2. 
Table 2: Tetranitro Zinc (II) Phthalocyanine 1 H NMR spectra data

\begin{tabular}{|c|c|c|}
\hline Shift (PPM) & Integration & Assignment \\
\hline 8.1335 & 0.4202 & Aggregated Aromatic C-H \\
\hline 7.2924 & 0.5733 & 2 Aromatic N-H \\
\hline 7.2812 & 1.2275 & 4 Aromatic C-H \\
\hline 7.1772 & 1 & 4 Aromatic C-H \\
\hline 7.0753 & 1.0618 & 4 Aromatic C-H \\
\hline
\end{tabular}

Table 2 indicates the formation of Tetranitro Zinc (II) Phthalocyanine, with distinctive 3 singlet aromatic peaks, 7.2812, 7.1772 and $7.0753(\mathrm{ppm})$, having integrations of $1.2275,1.0000$ and 1.0618 respectively.[11] Table 2 supports low aggregation sample formation, as an NMR peak was observed, at $8.1335 \mathrm{ppm}$, enjoying an integration of 0.420 and low integration value, due to downfield drifting.[12] NMR sample aggregation is far lower than that seen in Figure $1 \mathrm{UV}-$ Vis samples, due to the sample concentrations. $[5,6]$

\subsection{UV-Vis Results}

Figure 1 high aggregation UV-Vis spectra of Tetranitro Zinc (II) Phthalocyanine product was produced.

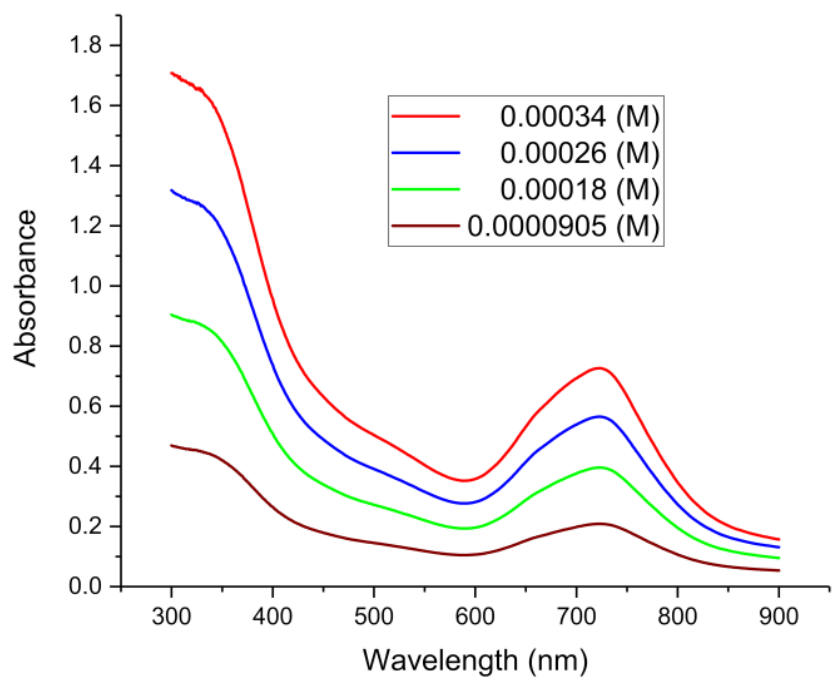

Figure 1: Tetranitro Zinc(II) Phthalocyanine experimental UV-Vis spectra at 0.00009011(M) 0.0001759(M) $0.0002577(M) \& 0.0003358(M)$

Analysis confirmed the formation of Tetranitro Zinc (II) Phthalocyanine, with both characteristic Q band in the $600-850(\mathrm{~nm})$ regions and Soret band in the $300-450(\mathrm{~nm})$ region are seen in figure 1. Literature reports of Phthalocyanine concentrations at $10^{-5}(\mathrm{M})$ and $10^{-4}(\mathrm{M})$, supported Tetranitro Zinc (II) Phthalocyanine aggregation occurring, in the UV-Vis sample, as indicated by the broadened and red-shifted spectra also seen in figure $1[5,6]$. With concentrations $>10^{-5}(\mathrm{M})$ reporting notably broad, red-shifted $\mathrm{Q}$ and Soret absorbance compared to ranges $<10^{-5}(\mathrm{M})$ distinctive of aggregation.[13]

\subsection{DFT Computational Results}

To confirm if aggregation was occurring, in figure 1, gas phase TD-DFT were computed calculating an ideal Soret range for gas phase Tetranitro Zinc (II) Phthalocyanine. Figure 2 displays the orbital configuration diagrams of HOMO-1, HOMO \& LUMO, Tetranitro Zinc (II) Phthalocyanine respectively. 

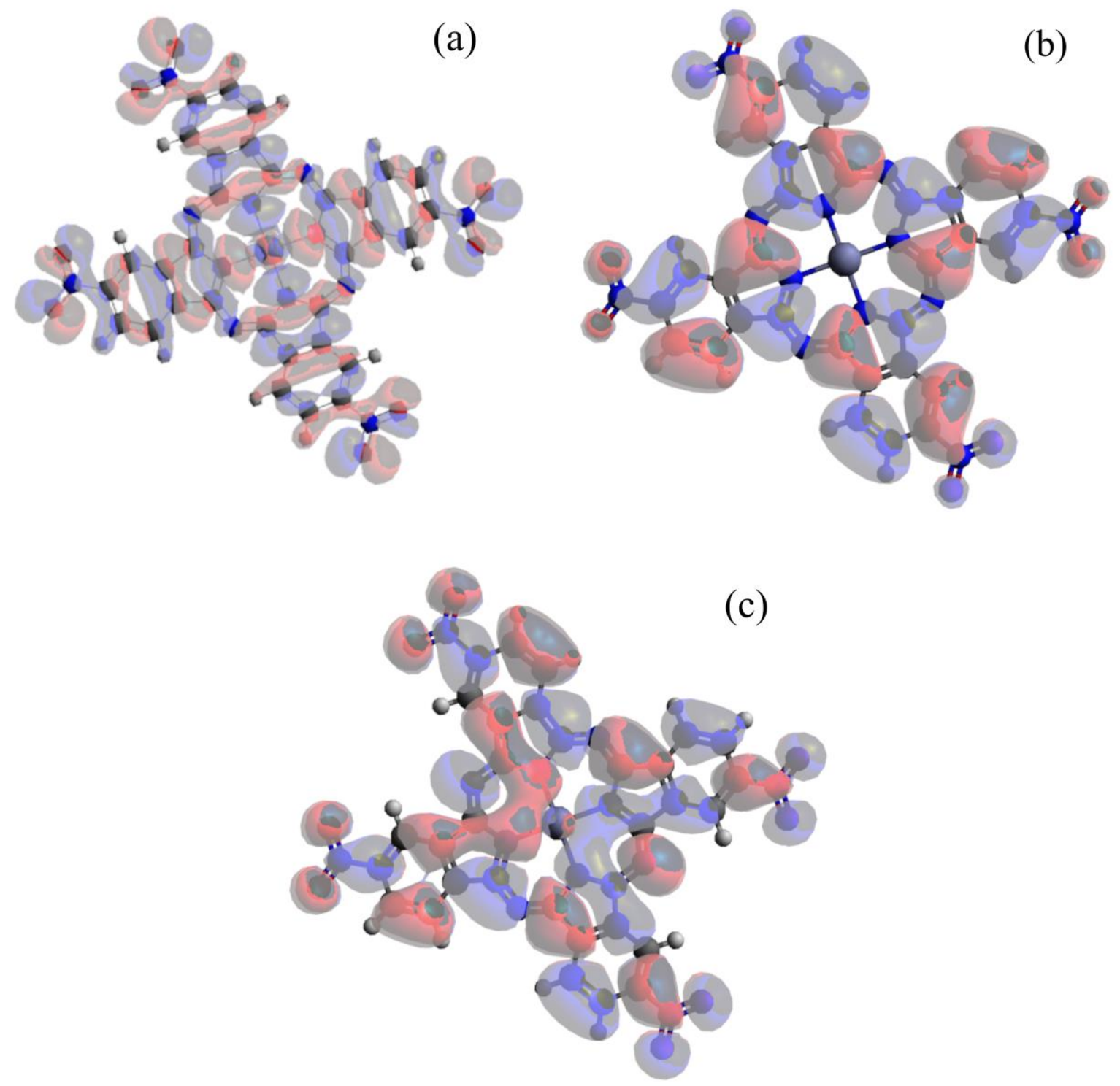

Figure 2: D4h Tetranitro Zinc (II) Phthalocyanine orbital diagrams of (a)HOMO-1 (b)HOMO \& (c)LUMO

TD-DFT computed ideal Soret absorbance region, corresponding to a $\pi$ HOMO- 1 to $\pi^{*}$ LUMO transition, for pure gas phase Tetranitro Zinc (II) Phthalocyanine ranged between 613-632(nm). TD-DFT computed Soret band absorbance range is supported by literature with absorbance reported between 610-690(nm) region [14,15]. The TD-DFT range isn't supported by the experimental data reported in figure 1 of between $600-800(\mathrm{~nm})$ and comparison of the two ranges is scientifically significant, with a $\mathrm{P}$ value of 0.047 . The discrepancy is believed to result from the TD-DFT computed results not accounting for aggregation, as Figure 2 demonstrates, only ideal gas phase Tetranitro Zinc (II) Phthalocyanine is computed.

\subsection{Tetranitro Zinc (II) Phthalocyanine Binding Mode}

NMR data in Table 2 and UV-Vis data in figure 1 both indicated aggregations was occurring, to varying degrees. $1 \mathrm{H}$ NMR data in table 2, experimentally indicated aggregation was occurring with downfield drifting theorised to occur from nitro to zinc electron donation.[12] UV-Vis data in figure 1, experimentally indicated aggregation was occurring with red shifting, compared to gas phase TD-DFT calculated ranges, 
Synthesis and Characterization of Zinc (II) Phthalocyanine for Screening Potential Solar Cell Dye Application

theorised to occur from Phthalocyanine J-type aggregation.[5,6] Thus the binding mode of Phthalocyanine was deduced as J-type aggregation resulting from nitro to zinc binding illustrated in figure 3.

\subsection{Tetranitro Zinc (II) Phthalocyanine Aggregation-Caused Quenching}

With the aggregation binding mode of Tetranitro Zinc (II) Phthalocyanine deduced, the computational DFT calculations of aggregated Tetranitro Zinc (II) Phthalocyanine could be completed to deduce if aggregation-caused quenching was occurring in Phthalocyanine as theorised or alternative energy transfer. Figure 3 illustrates the orbital diagrams of the expected Tetranitro Zinc (II) Phthalocyanine aggregated binding mode. DFT computational modelling of the Zinc (II) Phthalocyanine aggregated complex was completed, and confirmed theorised binding mode and supported aggregation-caused quenching theory.
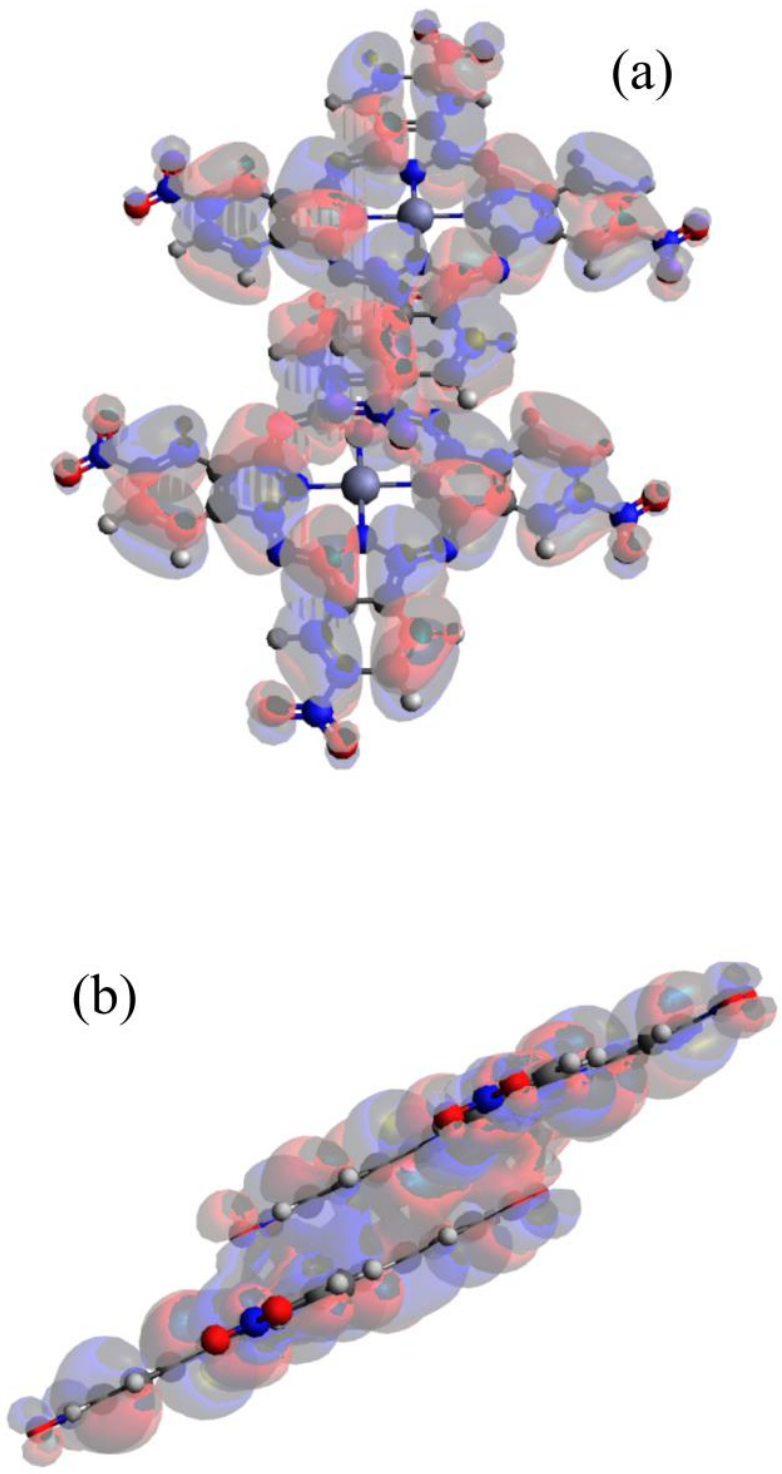

Figure 3: Phthalocyanine dimer complex (a)HOMO \& (b)LUMO orbital diagrams

Aggregation-Caused Quenching theory was supported by the Tetranitro to metal centre aggregation binding mode of Tetranitro Zinc (II) Phthalocyanine, allowing increased $\pi-\pi^{*}$ transitions between the complex aromatic units. Increased $\pi-\pi^{*}$ transitions influencing the sample UV-Vis via broadening of the absorbance ranges. Phthalocyanine J-type aggregation, has experimentally been shown to be somewhat favourable for 
use in solar cells although as previously mentioned any aggregation limits solar cell efficiency at generating useful electrical current [1,2].

\section{Conclusions}

Tetranitro Zinc (II) Phthalocyanine has successfully been synthesised via DBU catalysis, in $72 \%$ yield as confirmed via ATR-IR, 1H NMR and UV-Vis analysis of product. Tetranitro Zinc (II) Phthalocyanine UVVis analysis, of both Q \& Soret bands are notably broad and red shifted, characteristic of Phthalocyanine aggregation. UV-Vis and NMR aggregation data were used to deduce the type of aggregation occurring in the complex and J-type aggregation is indicated, previously reported to limit Phthalocyanine use in solar cells. J-aggregation is theorised to occur due to aggregation of the Phthalocyanine sample, resulting from Tetranitro-substituent and metal centre binding variations, allowing $\pi-\pi^{*}$ interactions between interacting $\mathrm{P}_{(z)}$ orbitals, supported by red-shifting and downfield drifting resulting from electron density donation to metal centre. Novel computational DFT calculations on the suspected J-type aggregation, binding mode of the sample seem to indicate the theorised aggregation-caused quenching theory is possible and occurring in the aggregated sample. This Novel approach potentially opens up the option for further screening of dye molecules aggregated binding mode in future studies to deduce if aggregation-caused quenching is occurring or a more desirable of energy transfer path. As this study has shown Tetranitro Zinc (II) Phthalocyanine has a strong tendency to aggregate, resulting in aggregation-caused quenching and thus leading to Phthalocyanine being ineffective at generating useful photo-charges, Tetranitro Zinc (II) Phthalocyanine is still recommended as undesirable as a solar organic semiconducting dye for use in future studies, at this concentration.

\section{Competing Interests}

The author declares that there is no conflict of interest that existed in the publication of the work.

\section{How to Cite this Article:}

C. Atkinson, "Synthesis and Characterization of Zinc (II) Phthalocyanine for Screening Potential Solar Cell Dye Application", J. Mod. Mater., vol. 9, no. 1, pp. 3-10, Jan. 2022.

\section{References}

[1] B. Yıldız, B. Seçkin Arslan, E. Güzel, M. Nebioğlu, N. Menges, İ. Şişman, M. Şener "Non-aggregating zinc phthalocyanine sensitizer with bulky diphenylphenoxy donor groups and pyrazole-3-carboxylic acid anchoring group for coadsorbent-free dye-sensitized solar cells", Solar Energy, 226, 7, September 2021, https://doi.org/10.1016/j.solener.2021.08.033

[2] C. Ilgün, A. M. Sevim, S. Çakar, M. Özacar, A. Gül, "Novel Co and Zn-Phthalocyanine dyes with octa-carboxylic acid substituents for DSSCs", Solar Energy 218, 7, April 2021, https://doi.org/10.1016/j.solener.2021.02.042

[3] G. Lyu, T. J. F. Southern, B. L. Charles, M. Roger, P. Gerbier, S. Clément and R. C. Evans, "Aggregation-induced emission from silole-based lumophores embedded in organic-inorganic hybrid hosts", J. Mater. Chem. C,9, 39, 2021. https://doi.org/10.1039/D1TC02794H

[4] Yu, M., Huang, R., Guo, J. et al. "Promising applications of aggregation-induced emission luminogens in organic optoelectronic devices." PhotoniX, 10, 1, November 2020. https://doi.org/10.1186/s43074-020-00012-y

[5] J. H. Kim, T. Schembri, D. Bialas, M. Stolte, F. Würthner, "Slip-Stacked J-Aggregate Materials for Organic Solar Cells and Photodetectors" Advanced Materials, 14, 3, October 2021. https://doi.org/10.1002/adma.202104678

[6] C. Ji, L. Lai, P. Li, Z. Wu, W. Cheng, M. Yin, "Organic dye assemblies with aggregation-induced photophysical changes and their bio-applications" Aggregate, 2, 4, February 2021. https://doi.org/10.1002/agt2.39

[7] M. A. Schmidt and J. F. Mário "Phthalocyanines: An Old Dog Can Still Have New (Photo)Tricks!", Molecules 26, 9, March 2021, https://doi.org/10.3390/molecules26092823

[8] Y, Okada, T. Hoshi and N. Kobayashi, "Recent Progress in Optically-Active Phthalocyanines and Their Related Azamacrocycles." Front. Chem. 8, 595998, October 2021. https://doi.org/10.3389/fchem.2020.595998

[9] E. H. Alici, A. T. Bilgiçli, A. Günsel, G. Arabaci and M. N. Yarasir, " $\alpha$-Substituted phthalocyanines based on metal-induced H- or Jtype aggregation for silver and palladium ions: synthesis, fluorescence, and antimicrobial and antioxidant properties" Dalton Trans.,9 ,50, January 2021, https://doi.org/10.1039/D0DT04103C

[10] R. Georgescu, C. Boscornea, I. Calinescu, R. State, "Raman, IR and UV-Vis spectroscopic investigations of some substituted phthalocyanines", Rev. CHIM. 10, 66, December 2015

[11] X. Zhang, X. Li, L. Niu, L. Sun and L. Liu, "Charge Transfer Photophysics of Tetra( $\alpha$-amino) Zinc Phthalocyanine", Journal of Fluorescence, 19, 947 December 2009, https://doi.org/10.1007/s10895-009-0494-7.

[12] V. K. Bind, "Supramolecular Phthalocyanine Aggregates", Indian Institute of Science Education and Research Master's thesis, June 2014 
Synthesis and Characterization of Zinc (II) Phthalocyanine for Screening Potential Solar Cell Dye Application

[13] Y. Wana, S. Chena, G. Wangb, Q. Lianga, Z. Lia,b \& S. Xu, "Facile Synthesis and Characterization of Zinc Tetranitro Phthalocyanine-MWCNTs Nanocomposites with Efficient Visible-Light-Driven Photocatalytic Activity", ACTA PHYSICA POLONICA, 3, 130, December 2016.

[14] M. Kareem, "Synthesis and Characterization of New Zinc-phthalocyanine with Four Dodecenyl-benzoic Pendant Groups", Pure and Applied Sciences, 2, 25, December 2017

[15] G. Martynov, J. Mack, K. May, "Methodological Survey of Simplified TD-DFT Methods for Fast and Accurate Interpretation of UVVis-NIR Spectra of Phthalocyanines", ACS Omega., 4, 14, April 2019, https://doi.org/10.1021/acsomega.8b03500

Publish your research article in AIJR journals-

* Online Submission and Tracking

* Peer-Reviewed

* Rapid decision

* Immediate Publication after acceptance

* Articles freely available online

* Retain full copyright of your article.

Submit your article at journals.aijr.org
Publish your books with AIJR publisher-

* Publish with ISBN and DOI.

* Publish Thesis/Dissertation as Monograph.

* Publish Book Monograph.

* Publish Edited Volume/ Book.

* Publish Conference Proceedings

* Retain full copyright of your books.

Submit your manuscript at books.aijr.org 